\title{
The Pharmacist as Part of the Primary Care Team During the COVID-19 Pandemic
}

\author{
Cynthia Moreau, PharmD, BCACP
}

Pharmacists' roles and training have evolved to prepare pharmacists to provide clinical patient care services as part of interdisciplinary teams in primary care settings. Especially now, amidst a global health crisis such as COVID-19, patients may become more aware of their health status and be exposed to increased medical information in the media. Additionally, some patients may have delayed routine care, which may result in exacerbations of chronic disease states. Pharmacists can help alleviate the burden on primary care providers by serving as a drug information resource for patients and staff while providing patient education on management of chronic disease states. ( $\mathrm{J}$ Am Board Fam Med 2021;34:S21-S25.)

Keywords: Chronic Disease, CoVID-19, Delivery of Health Care, Patient Care, Pharmacists, Primary Health Care

The Association of American Medical Colleges (AAMC) estimates that by the year 2032, there will be a shortage of 21,100 to 55,200 primary care physicians in the United States. According to AAMC, addressing this shortage requires a multipronged approach, including efficient use of health professionals on the care team. ${ }^{1}$ Pharmacists are health professionals that can be utilized to ensure patients receive adequate care in primary care settings.

The traditional role of the pharmacist has evolved such that pharmacy graduates are being trained to provide patient care services that are not directly associated with medication dispensing, including interprofessional, collaborative medication management services such as medication therapy management (MTM) or comprehensive medication management $(\mathrm{CMM})^{2,3}$ In fact, the American Association of Colleges of Pharmacy (AACP) is aiming for at least $50 \%$ of primary care medical practices to have pharmacist-provided CMM services integrated into their care models by the year 2025..$^{4}$ Furthermore, the American Medical Association (AMA) has provided

From the Department of Pharmacy Practice, Nova Southeastern University College of Pharmacy, Fort Lauderdale, FL (CM).

Disclosure statement: The author has no disclosures or conflicts of interest.

Corresponding author: Cynthia Moreau, PharmD, BCACP, Nova Southeastern University College of Pharmacy, 3200 S University Drive, Fort Lauderdale, FL 33328, Phone: 954593-9913 (E-mail: cm1524@nova.edu). direction to providers on how to embed pharmacists into practice to improve patient outcomes. ${ }^{5}$

Despite these initiatives, it seems pharmacists have not yet been fully incorporated as part of primary care practice teams. A survey of over 11,000 family physicians in 2016 revealed that pharmacists were present in fewer than $20 \%$ of family physician practice teams. ${ }^{6}$

\section{What Can a Pharmacist Do for You? Pharmacist Education and Training}

Pharmacists are highly trained medication experts. A traditional Doctor of Pharmacy (PharmD) program consists of a 4-year curriculum, including 3 years of didactic education followed by 1 year dedicated to clinical clerkships. Many pharmacy graduates also seek advanced clinical training through 1 or 2 years of postgraduate residency training. Generally, a postgraduate year 1 (PGY1) residency provides advanced general training while postgraduate year 2 (PGY2) residency programs are more specialized in 1 area of practice. Specifically, PGY2 ambulatory care pharmacy residencies provide advanced training to create clinical pharmacy practitioners that can manage patients with a variety of chronic disease states. Compared with other available PGY2 residency opportunities, ambulatory care pharmacy residency positions have grown exponentially in recent years, ${ }^{7}$ demonstrating an increased supply of pharmacists that are trained to practice within primary care 
settings. Even without the completion of postgraduate training, pharmacists may have other credentials that allow them to care for patients with chronic disease states. The Board of Pharmacy Specialties (BPS) establishes criteria for pharmacists to earn certification in various practice areas including ambulatory care practice (BCACP), pharmacotherapy (BCPS), geriatric pharmacy (BCGP), and cardiology pharmacy (BCCP), among other specialties. ${ }^{8}$ In addition, state-specific legislation may expand pharmacists' scope of practice so they may enter into collaborative practice agreements (CPAs) and provide clinical services under the supervision of a licensed physician. ${ }^{9,10}$

\section{Patient Care Services That Can Be Provided by Pharmacists}

Pharmacists can bring value to primary care practice teams through a variety of activities aimed to optimize medication use. High rates of chronic disease in the United States have contributed to increasing prevalence of polypharmacy. ${ }^{11}$ Prescribing medications in this context can be complicated for providers, due to increased potential for drug interactions, adverse drug reactions, and potentially inappropriate prescribing, such as under- or overprescribing. ${ }^{12}$

Published literature evaluating the effectiveness of pharmacist services in primary care practice has demonstrated that pharmacists can improve management of chronic conditions as well as the quality of prescribing and medication appropriateness. ${ }^{13}$ As each practice team has unique medication-related needs and patient populations, it is important for them to evaluate how pharmacist contributions can add value to the practice. ${ }^{14}$ Providers can begin by prioritizing patients who could most benefit from pharmacist services, which include those with uncontrolled chronic disease states and those with polypharmacy issues. ${ }^{4}$ In addition, teams may want to consider how pharmacists can help them attain quality measures, such as those delineated by the Centers for Medicare \& Medicaid Services (CMS). ${ }^{15-17}$ In a study by Brummel and colleagues evaluating pharmacist-led MTM services for complex patients with diabetes within an accountable care organization (ACO), patients who received pharmacist care were more likely to achieve better diabetes control as measured by changes in hemoglobin A1c, blood pressure, lowdensity lipoprotein levels, aspirin use, and no smoking. ${ }^{18}$ Quality metrics related to diabetes control, statin use, hypertension control, and tobacco use are currently included as part of the Medicare Shared Savings Program Quality Measure Benchmarks. ${ }^{17}$

Pharmacists may also assist practices with delivery of clinical services to potentially increase practice revenue such as chronic care management (CCM), transitional care management (TCM), and annual wellness visits (AWVs). In collaboration with providers, pharmacists can act as clinical staff to complete some components required to bill for these services (eg, post-discharge telephone call for TCM, medication reconciliation, and management for CCM). ${ }^{19-21}$ Finally, pharmacists can assist with delivery of MTM services required as part of the Medicare Part D Medication Therapy Management program. $^{22}$

\section{Provider Perceptions of Pharmacist Integration}

The primary care physician shortage along with an evolving health care landscape can lead to burnout among providers. It has been estimated that over half of physicians are experiencing burnout, with highest rates among family medicine and internal medicine practitioners. ${ }^{23}$ Studies assessing provider perceptions of pharmacist integration in primary care settings show that most providers felt that pharmacists are valuable members of the team and increased efficiency of patient care by allowing them to focus on medical concerns while pharmacists managed medicationrelated problems. ${ }^{23-25}$ Satisfaction with pharmacist integration also extended beyond providers in a study by Kozminski et al, in which nonclinical office staff felt that pharmacists provided a valuable service to patients and served as a drug information resource for staff. ${ }^{24}$

\section{Why Now?}

Pharmacy education and training have evolved to prepare pharmacists to provide patient care services to support the interprofessional team in primary care settings. In addition, pharmacists have long been recognized as 1 of the most accessible and frequently visited health care professionals. In recent months, the coronavirus pandemic has significantly affected individuals in the United States and brought our nation's health care industry and workers into the national spotlight. Pharmacists are essential in caring for patients affected by Coronavirus disease 2019 (COVID-19) across a variety of health care settings. Notably, in April 2020, the US Department of Health and Human Services 
authorized licensed pharmacists to order and administer COVID-19 tests approved by the US Food and Drug Administration. ${ }^{26}$

During a global health crisis, patients have many questions regarding their health, medications, and immunization status. In the case of COVID-19, patients have access to an abundance of information through the media regarding potential treatments for the virus. For example, some reports raised potential safety concerns regarding the use of nonsteroidal anti-inflammatory drugs (NSAIDs) and angiotensin converting enzyme (ACE) inhibitors. ${ }^{27,28}$ In addition, medications such as hydroxychloroquine, azithromycin, remdesivir, and famotidine have been reported as investigational treatments for the disease. ${ }^{29,30}$ Pharmacists on care teams can help alleviate the burden on providers to answer patient questions regarding these topics by serving as a resource for patients and staff during times of crises while helping to maintain continuity of care for patients. For example, during the pandemic, pharmacists at the University of Florida supported physicians by triaging patients telephonically and scheduling telehealth appointments with physicians. ${ }^{31}$ At Livingston HealthCare hospital in Montana, pharmacists assisted with driveup anticoagulation services to ensure patients were receiving regular international normalized ratio (INR) monitoring while minimizing the potential exposure risk associated with entering the hospital. ${ }^{32}$ These serve as examples of services that could also be provided by a pharmacist in a primary care setting.

Furthermore, as a result of COVID-19, the delivery of health care in primary care settings has significantly changed. Providers may defer preventive visits or convert to telemedicine visits. Patients may also avoid visits due to fear of being exposed. An April 2020 report showed that the number of visits to ambulatory practices decreased by nearly $60 \%$ in mid-March 2020 and remained low through mid-April 2020. ${ }^{33}$ As a result of patients not receiving routine care, it is possible that primary care providers may observe an increased rate of poor control and/or exacerbations of chronic disease states as patients return to primary care offices in the future. Patients who may not have been able to obtain essential chronic medications from their pharmacies or who had safety concerns related to their medications (ie, ACE inhibitors) may be especially at risk of having worse outcomes due to poor medication adherence. As patients begin returning to primary care settings, pharmacists can be utilized to address gaps in care that may have occurred during the pandemic as well as to provide patient education on how to manage chronic disease states and medication regimens if regular in-clinic follow-up cannot be achieved. As CMS loosened restrictions related to the delivery of telehealth services because of the pandemic, pharmacists can deliver these clinical services and provide patient follow-up through telehealth modalities that can be billed incident-to a licensed physician. ${ }^{34}$

\section{Barriers to Pharmacist Integration}

One of the biggest challenges to integration of pharmacists in primary care settings is sustainable funding to support pharmacist salaries. Pharmacists may bill for some services through incident-to billing, however, revenue generated solely from this is unlikely to support a pharmacist salary. ${ }^{35}$ Partnerships with colleges and schools of pharmacy may be developed to allow clinical pharmacy faculty and trainees to integrate within primary care clinics and pilot pharmacy-led services. ${ }^{36}$ Primary care practice groups may also consider hiring 1 or a few pharmacists that can provide services within multiple offices on various days of the week. ${ }^{25}$ Cost savings to the practices as a result of implementing pharmacy services may justify and support the addition of a pharmacist to the staff. In the study by Brummel and colleagues, a pharmacist-led MTM program resulted in an estimated 12:1 return on investment (ROI) during a 1-year period when comparing health care costs of patients receiving MTM services when matched to patients who did not receive these services. In addition, total annual health expenditures decreased from $\$ 11,965$ to $\$ 8,197$ per person. ${ }^{18}$ Finally, practices may consider developing partnerships and business agreements with local community pharmacies such that pharmacists can deliver remote and/or on-demand clinical services. ${ }^{5}$ This may be a more cost-effective option for practices and an opportunity to pilot pharmacy services on a smaller scale, especially during the COVID-19 pandemic when practices may not be able to justify additional spending to hire a pharmacist.

In conclusion, during these difficult times and beyond, pharmacists can be essential as members of the primary care team to support the provision of optimal patient care through disease state education, 
medication optimization, and medication adherence counseling. Pharmacists are ready to help you.

To see this article online, please go to: http://jabfm.org/content/ 34/Supplement/S21.full.

\section{References}

1. Association of American Medical Colleges. Physician supply and demand a 15-year outlook: key findings. 2019. Available at: https://www.aamc.org/system/files/ 2019-07/workforce_projections-15-year_outlook_-key_ findings.pdf. Accessed on April 17, 2020.

2. Urick BY, Meggs EV. Towards a greater professional standing: evolution of pharmacy practice and education, 1920-2020. Pharmacy 2019;7:98.

3. Joint Commission of Pharmacy Practitioners. Medication management services (MMS) definition and key points. Available at: https://jcpp.net/wpcontent/uploads/2018/05/Medication-ManagementServices-Definition-and-Key-Points-Version-1.pdf. Published March 2018. Accessed July 15, 2020.

4. Maine LL. Publisher's note. Academic Pharmacy Now 2019;4. Available at: https://issuu.com/aacp/ docs/apn-2019issue4. Accessed April 24, 2020.

5. Choe HM, Standiford CJ, Brown MT. Embedding pharmacists into the practice. AMA STEPS Forward. 2018. Available at: https://edhub.ama-assn.org/stepsforward/module/2702554. Accessed April 24, 2020.

6. Bazemore A, Wingrove P, Peterson L, Patterson S. The diversity of providers on the family medicine team. J Am Board Fam Med 2016;29:8-9. doi: 10.3122/jabfm.2016.01.150229.

7. American Society of Health-System Pharmacists. Residency match phase i signals steady growth in positions. 2020. Available at: https://www.ashp.org/ News/2020/03/13/ASHP-2020-Residency-MatchPhase-I-Signals-Steady-Growth-in-Positions. Accessed April 24, 2020.

8. Board of Pharmacy Specialties. Available at: https:// www.bpsweb.org/. Accessed April 24, 2020.

9. North Carolina Board of Pharmacy. Clinical pharmacist practitioners. Available at: http://www.ncbop.org/ pharmacists_cpp.htm. Accessed July 14, 2020.

10. Florida Board of Pharmacy. New legislation impacting your profession. Available at: https://floridaspharmacy. gov/latest-news/new-legislation-impacting-yourprofession-5/. Accessed July 14, 2020.

11. Quinn KJ, Shah NH. A dataset quantifying polypharmacy in the United States. Sci Data 2017;4:170167. doi:10.1038/sdata.2017.167.

12. Cardwell K, Smith SM. Clinical pharmacists working within family practice: What is the evidence? Fam Pract 2018;35:120-1.

13. Tan ECK, Stewart K, Elliot RA, George J. Pharmacist services provided in general practice clinics: A systematic review and meta-analysis. Res Social Adm Pharm 2014;10:608-22.
14. Jorgenson D, Dalton D, Farrell B, Tsuyuki RT, Dalovich L. Guidelines for pharmacists integrating into primary care teams. Can Pharm J (Ott) 2013;146: 342-52.

15. Centers for Medicare \& Medicaid Services (CMS). Quality measures. Available at: https://www.cms.gov/ Medicare/Quality-Initiatives-Patient-AssessmentInstruments/QualityMeasures. Accessed April 27, 2020.

16. Quality Payment Program. Quality measures requirements. Available at: https://qpp.cms.gov/ mips/quality-measures. Accessed April 27, 2020.

17. Centers for Medicare \& Medicaid Services (CMS). Medicare shared savings program quality measure benchmarks for the 2020/2021 performance years. Available at: https://www.cms.gov/files/document/2020 2021-quality-benchmarks.pdf. Accessed July 15, 2020.

18. Brummel A, Lustig A, Westrich K, et al,. Best practices: improving patient outcomes and costs in an ACO through comprehensive medication therapy management. JMCP 2014;20:1152-8.

19. American Society of Health-System Pharmacists. FAQ: Medicare annual wellness visits. Available at: https:// www.ashp.org/-/media/assets/pharmacy-practice/ resource-centers/ambulatory-care/medicare-annualwellness-visits.ashx. Published June 2018. Accessed July 14, 2020.

20. American Society of Health-System Pharmacists. Billing for transitional care management services. Available at: https://www.ashp.org/-/media/assets/ pharmacy-practice/resource-centers/ambulatory-care/ transitional-care-management-codes.ashx?la=en\&hash= 668D50A1EC1C57494BF81333FB63402A5C12A325. Published June 2019. Accessed July 14, 2020.

21. American Society of Health-System Pharmacists. FAQ: chronic care management. Available at: https:// www.ashp.org/-/media/assets/pharmacy-practice/ resource-centers/ambulatory-care/chronic-caremanagement-faq.ashx? la=en\&hash=A24F2B9E9B BE4C31E0361111EFD8CA61ADCAC211. Published July 2018. Accessed July 14, 2020.

22. Centers for Medicare \& Medicaid Services. Medication therapy management. Available at: https://www.cms.gov/Medicare/Prescription-DrugCoverage/PrescriptionDrugCovContra/MTM. Accessed July 14, 2020.

23. Funk KA, Pestka DL, Roth McClurg MT, Carroll JK, Sorensen TD. Primary care providers believe that comprehensive medication management improvements their work-life. J Am Board Fam Med 2019;32:462-73.

24. Kozminski M, Busby RS, McGivney M, Klatt PM, Hackett SR, Merenstein JH. Pharmacist integration into the medical home: qualitative analysis. J Am Pharm Assoc (2003) 2011;51:173-83.

25. Moreno G, Lonowski S, Fu J, et al. Physician experiences with clinical pharmacists in primary care teams. J Am Pharm Assoc (2003) 2017;57:686-91. 
26. US Department of Health \& Human Services. HHS statements on authorizing licensed pharmacists to order and administer COVID-19 tests. Available at: https://www.hhs.gov/about/news/2020/04/08/hhsstatements-on-authorizing-licensed-pharmacists-toorder-and-administer-covid-19-tests.html. Accessed April 27, 2020.

27. US Food and Drug Administration. FDA advises patients on the use of non-steroidal anti-inflammatory drugs (NSAIDs) for COVID-19. Available at: https:// www.fda.gov/drugs/drug-safety-and-availability/fdaadvises-patients-use-non-steroidal-anti-inflammatorydrugs-nsaids-covid-19. Accessed April 27, 2020.

28. Murray S. New evidence concerning safety of ACE Inhibitors, ARBs in COVID-19. Pharmacy Times. 2020. Available at: https://www.pharmacytimes. $\mathrm{com} /$ news/new-evidence-concerning-safety-of-aceinhibitors-arbs-in-covid-19. Accessed April 28, 2020.

29. National Institutes of Health. COVID-19 treatment guidelines. Available at: https://www.covid19treatmentguide lines.nih.gov/introduction/. Accessed April 28, 2020.

30. Ientile GF. Trial underway in NYC for COVID-19 treatment. Drug Topics. 2020. Available at: https:// www.drugtopics.com/latest/famotidine-trial-underwaynyc-covid-19-treatment. Accessed April 28, 2020.

31. University of Florida College of Pharmacy. Answering the call: UF College of Pharmacy responds with call center support during pandemic. Available at: https:// pharmacy.ufl.edu/2020/04/21/answering-the-calluf-college-of-pharmacy-responds-with-call-center- support-during-pandemic/. Published April 2020. Accessed July 14, 2020.

32. Traynor K. American Society of Health-System Pharmacists. Pandemic prompts switch to drive-up anticoagulation testing. Available at: https://www.ashp. org/News/2020/03/27/Pandemic-Prompts-Switch-toDrive-Up-Anticoagulation-Testing?loginreturnUrl= SSOCheckOnly. Published March 2020. Accessed July 14, 2020.

33. Mehrota A, Chernew M, Linetsky D, Hatch H, Cutler D. The Impact of the COVID-19 Pandemic on Outpatient Visits: A Rebound Emerges. 2020. Available at: https://www.commonwealthfund.org/ publications/2020/apr/impact-covid-19-outpatientvisits. Accessed December 30, 2020.

34. American Society of Health-System Pharmacists. Issue brief: COVID-19 and telehealth changes. Available at: https://www.ashp.org/Advocacy-andIssues/Key-Issues/Other-Issues/Issue-Brief-COVID19? loginreturnUrl=SSOCheckOnly. Published April 2020. Accessed July 14, 2020.

35. Dietrich E, Gums JG. Incident-to billing for pharmacists. J Manag Care Spec Pharm 2018;24: 1273-6.

36. Joseph T, Hale GM, Eltaki SM, et al. Integration strategies of pharmacists in primary care-based accountable care organizations: a report from the Accountable Care Organization Research Network, Services, and Education. J Manag Care Spec Pharm 2017;23:541-8. 